\title{
Granulomatous Hepatitis Secondary to Histoplasmosis in an Immunocompetent Patient
}

\author{
Adel Muhanna ${ }^{1}$, Faisal M. Nimri ${ }^{2}$, Zaid A. Almomani ${ }^{3}$, Laith Al Momani ${ }^{4}$, Alisa Likhitsup ${ }^{5}$ \\ 1. Internal Medicine, University of Missouri Kansas City, Kansas City, USA 2. Internal Medicine, Henry Ford Health \\ System, Detroit, USA 3. Internal Medicine, Jordan University of Science and Technology, Irbid, JOR 4. \\ Gastroenterology, University of Missouri Kansas City, Kansas City, USA 5. Gastroenterology and Hepatology, \\ University of Missouri Kansas City, Kansas City, USA
}

Corresponding author: Alisa Likhitsup, alikhitsup@gmail.com

\begin{abstract}
Histoplasma capsulatum is the most common endemic mycosis in the United States and usually occurs in certain geographic areas, such as the Mississippi or Ohio River valleys. Histoplasmosis usually causes a mild disease in the immunocompetent but can progress to disseminated disease in patients with impaired immunity. Granulomatous hepatitis as a manifestation of disseminated histoplasmosis in immunocompetent patients is extremely rare. We report the case of a 62 -year-old immunocompetent gentleman with a history of histoplasmosis who presented with abdominal pain, elevated liver enzymes, who was diagnosed with granulomatous hepatitis secondary to histoplasmosis.
\end{abstract}

Review began 08/01/2021 Review ended 08/25/2021 Published 09/01/2021

\section{๑) Copyright 2021}

Muhanna et al. This is an open access article distributed under the terms of the Creative Commons Attribution License CC-BY 4.0., which permits unrestricted use, distribution, and reproduction in any medium, provided the original author and source are credited.
Categories: Pathology, Gastroenterology, Infectious Disease

Keywords: endemic mycosis, granulomatous hepatitis, gastrointestinal histoplasmosis, elevated liver enzyme, immuno-competent

\section{Introduction}

Histoplasmosis, caused by Histoplasma capsulatum var. capsulatum, is currently the most common endemic mycosis in the United States (US), with an estimated incidence of 500,000 cases annually in the US [1]. Although histoplasmosis occurs primarily in the Midwestern and Central areas of the US, especially along the Mississippi and Ohio River Valleys, these infections can be found in other areas across the country $[2,3]$. While most patients infected with histoplasmosis have asymptomatic or mild disease, around 5\% of patients develop symptomatic pulmonary infections that can progress to severe disseminated infection in patients with compromised immunity [4]. Gastrointestinal manifestations are found in less than $10 \%$ of patients, with liver involvement being even less common [5]. Herein, we present the case of a 62 -year-old immunocompetent man who presented with transaminitis secondary to histoplasmosis.

\section{Case Presentation}

A 62-year-old gentleman with significant past medical history of gastroesophageal reflux disease who presented to the emergency department with right upper quadrant abdominal pain, associated with nausea, low appetite and loose stool for one month prior to his presentation. He denies any fever or chills. His medications include proton pump inhibitors for his acid reflux otherwise denied history of new medication. He is a retired mailman who did not have any house pet. There was no recent travel history or sick exposure. The patient is a current cigarette smoker, and he denies alcohol consumption or illicit drug use. Physical exam revealed afebrile, mild sinus tachycardia, a well-appearing alert and oriented male without jaundice yet had prominent signs of chronic pruritus. Laboratory tests revealed unremarkable basic metabolic panel, aspartate transaminase (AST) of $321 \mathrm{IU} / \mathrm{L}$, alanine aminotransferase (ALT) of $228 \mathrm{IU} / \mathrm{L}$, alkaline phosphate (ALP) $462 \mathrm{IU} / \mathrm{L}$, total bilirubin of $2.1 \mathrm{mg} / \mathrm{dl}$, direct bilirubin $0.2 \mathrm{mg} / \mathrm{dL}$, white blood cells (WBC) of $4.30 \mathrm{~K} / \mathrm{ml}$, platelets of $148 \mathrm{~K} / \mathrm{ml}$, and an international normalized ratio (INR) of 1.1. The patient's work was negative for acute and chronic viral hepatitis, human immunodeficiency virus, cytomegalovirus. Chronic liver disease markers including antinuclear antibodies, anti-smooth muscle antibodies, anti-mitochondrial antibodies, ceruloplasmin, alpha-1 antitrypsin, and iron studies were unremarkable. Magnetic Resonance Imaging (MRI) of the abdomen showed a normal liver, bilateral adrenal masses (Figures 1,2), splenomegaly and incidental moderate gallbladder wall thickening without gallstones. Ultrasonography (USG) of the abdomen was suboptimal and decision was made to get Endoscopic Ultrasonography (EUS) which showed findings of gallbladder sludge and fatty liver. Cholecystectomy was recommended to address patient symptoms and gallbladder sludge with concurrent liver biopsy to evaluate elevated liver function tests (LFTs). Of note, Computed Tomography (CT) scan of the chest was done and it showed no lung lesions. The patient underwent laparoscopic cholecystectomy with liver biopsy. The pathology showed chronic cholecystitis and liver biopsy showed marked bile duct reaction with reactive changes consisting of portal tract inflammatory cell infiltration, with a foci of lymphocytic cholangitis as well as a few lymphocytes in sinusoids, portal granuloma with giant cells, and fibrotic changes in the liver (Figure 3). Grocott's methenamine silver (GMS) stain showed scattered fungal yeast forms within portal areas in association with portal-based granulomas, morphologically consistent with Histoplasma species (Figure 4). Acid-fast bacilli staining was negative for 


\section{Cureus}

acid-fast organisms. These histologic findings were consistent with granulomatous hepatitis secondary to histoplasmosis. The patient was started on daily treatment of itraconazole followed by regular follow-ups. The patient's right upper quadrant abdominal pain has resolved after cholecystectomy and his laboratory follow-up results have normalized. His most recent LFTs were obtained 9 months from starting itraconazole and showed an ALT of $16 \mathrm{IU} / \mathrm{L}$, AST of $24 \mathrm{IU} / \mathrm{L}$, ALP of $101 \mathrm{IU} / \mathrm{L}$, total bilirubin of $1.2 \mathrm{mg} / \mathrm{dl}$, and direct bilirubin of $0 \mathrm{mg} / \mathrm{dl}$.

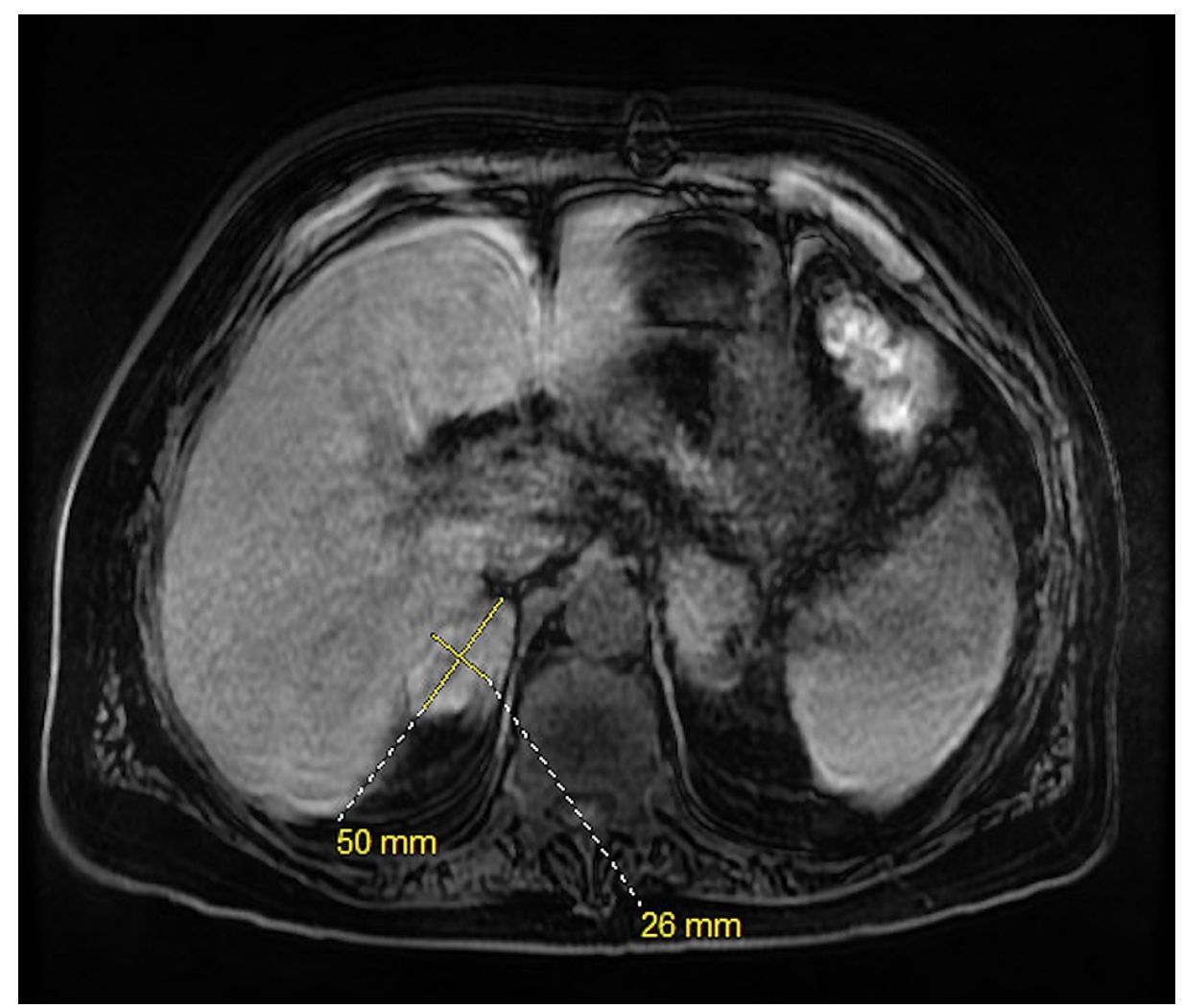

FIGURE 1: Transversal T1-weighted magnetic resonance image of the abdomen after contrast

Transversal T1-weighted magnetic resonance image of the abdomen after contrast showing right adrenal mass measuring $5 \mathrm{~cm} \times 2.6 \mathrm{~cm}$ 


\section{Cureus}

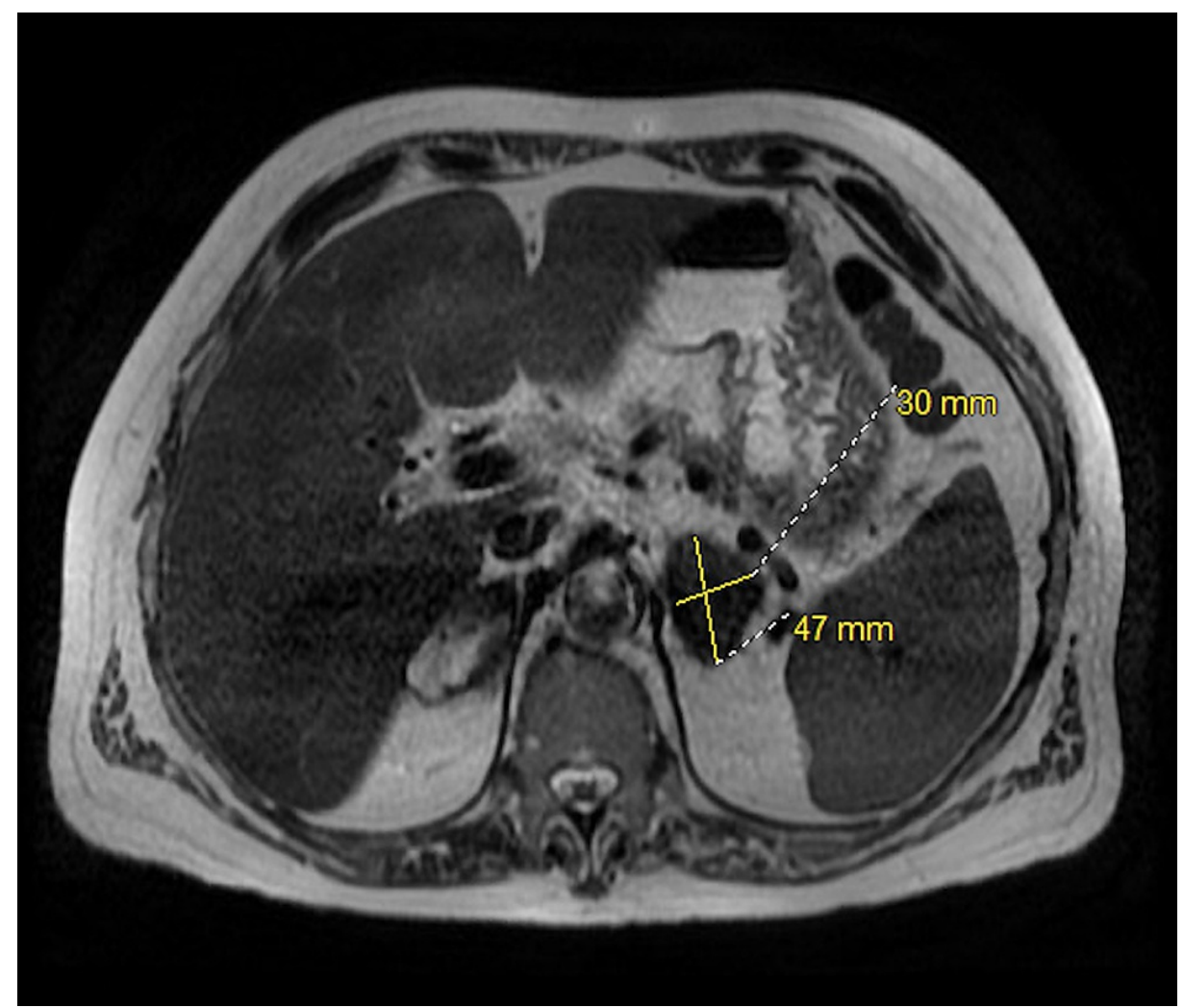

FIGURE 2: Transversal T1-weighted image of the abdomen without contrast

Transversal T1-weighted image of the abdomen without contrast showing left adrenal mass measuring 4.7 $\mathrm{cm} \times 3.0 \mathrm{~cm}$ 


\section{Cureus}

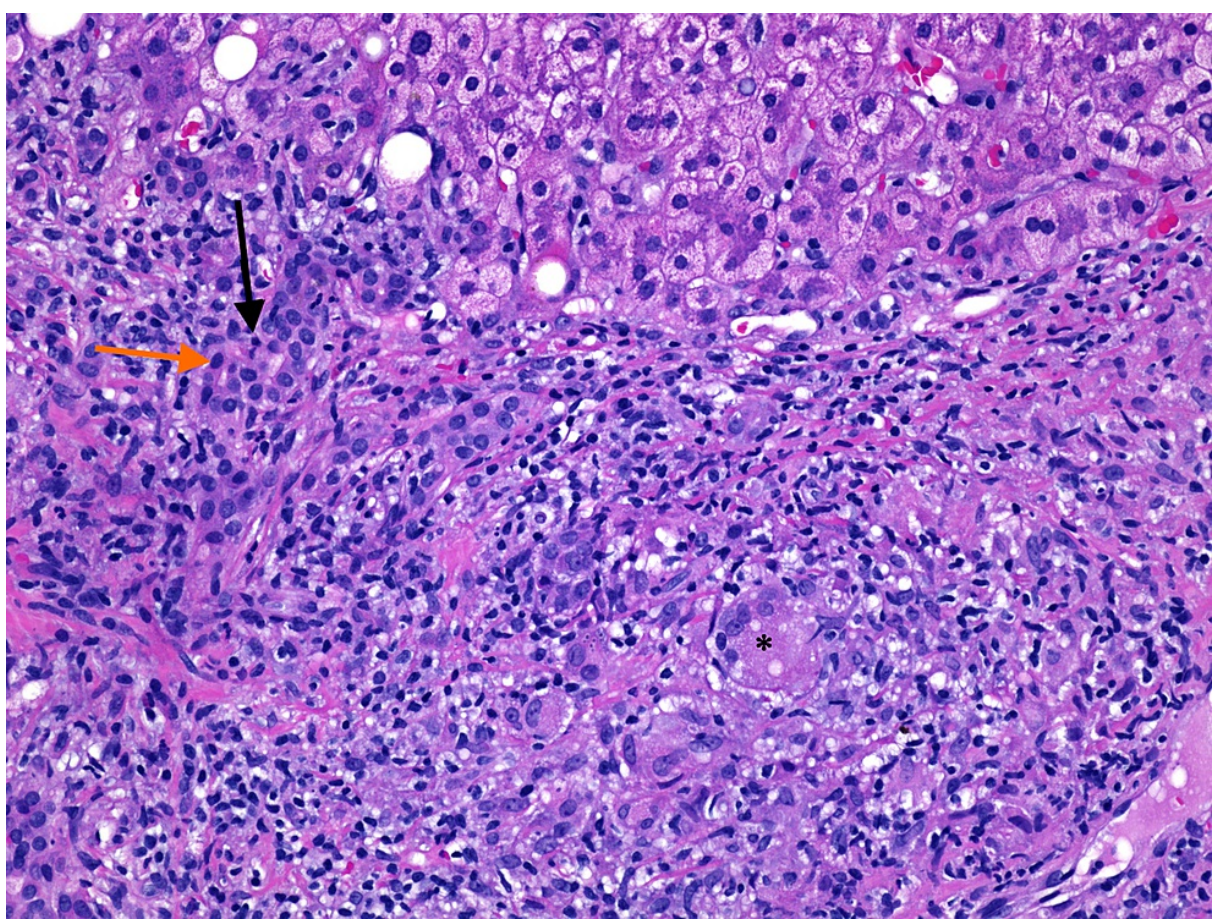

FIGURE 3: Hematoxylin and Eosin (H\&E) stain of liver biopsy (H\&E stain 20x).

Marked bile duct reaction (black arrow) with reactive changes, foci of lymphocytic cholangitis (orange arrow), and portal granuloma with giant cells (asterisk).

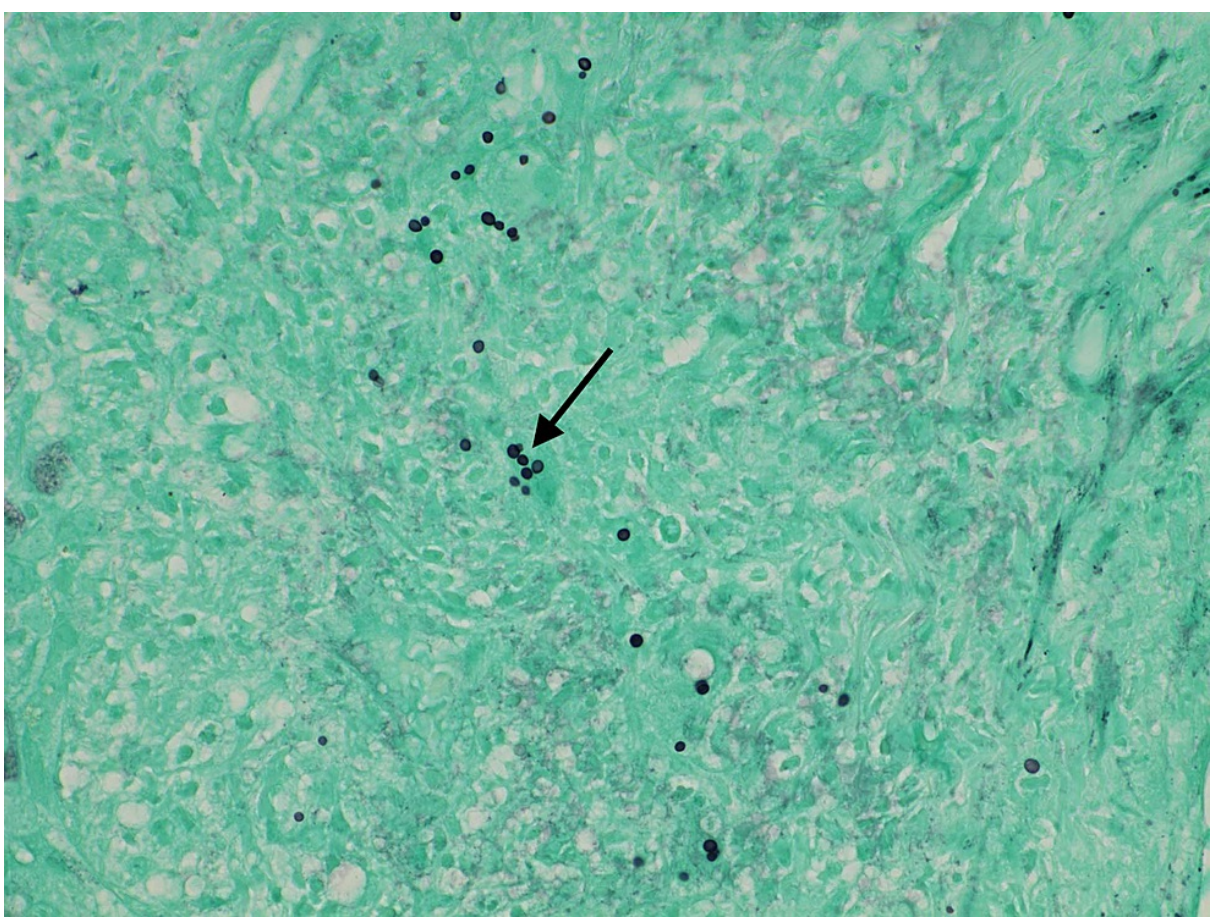

FIGURE 4: Grocott's methenamine silver (GMS) stain of liver biopsy (GMS stain 40x).

Scattered fungal yeast forms presented within portal areas in association with portal-based granulomas morphologically consistent with Histoplasma species. 


\section{Discussion}

Histoplasma capsulatum is a thermally dimorphic fungus, found as a mold in the environment and as a yeast at $37^{\circ} \mathrm{C}[6]$. Infection develops when the spores are inhaled into the lungs. Histoplasmosis is classified into pulmonary and extrapulmonary or disseminated histoplasmosis (DH). Cell-mediated immunity plays the most important role in recovery from histoplasmosis, either by killing the organism or by forming a calcified granuloma [7], and failure to achieve cell-mediated immunity is one of the key defects in patients with DH. Unlike in our case, DH is mostly found in immunocompromised patients, such as those with AIDS, primary immunodeficiencies, and solid organ transplant recipients [8].

The presentation of histoplasmosis in symptomatic patients most commonly resembles community-acquired pneumonia and usually consists of mild to moderate symptoms of cough, fever, dyspnea, headache, and arthralgia. However, the clinical presentation of DH can differ between individuals, and patients with acute infection can present with fatigue, fever, pancytopenia or coagulopathy, and hepatosplenomaegaly [3]. Adrenal involvement occurs in approximately $80 \%$ of patients with DH but adrenal insufficiency is found in the minority of cases [9], which may explain our patient's splenomegaly bilateral adrenal assess on CT imaging. Even though gastrointestinal involvement is common in DH, gastrointestinal disease is rarely identified and causes clinical symptoms only in 3 to $12 \%$ of patients [10]. The most common gastrointestinal findings include mucosal ulcerations that can be located anywhere from the mouth to the anus, colonic perforation, and masses that may resemble malignancies [11]. Liver involvement in histoplasmosis is even less commonly reported. Granulomatous hepatitis has a wide range of differential diagnoses including autoimmune, drug-related, infectious, a manifestation of systematic diseases, and idiopathic [12]. Therefore, the diagnosis and management can be challenging, and physicians must have a high index of suspicion because of the high fatality in patients with DH if left untreated.

To our knowledge, there are very few cases in the literature describing granulomatous hepatitis due to histoplasmosis, especially in immunocompetent adults [13-18]. One case reported a previously healthy man who presented with fever, hepatomegaly, and anorexia and was found to have granulomatous hepatitis due to acute histoplasmosis [13]. Another case was that of a 66-year-old female who presented with fever of unknown origin and an elevation in her liver enzymes as a primary presentation of histoplasmosis. However, unlike our case, the patient had a past medical history of cell-mediated immunodeficiency, a known risk factor for DH [14]. The diagnosis in both cases was established by tissue sampling. Despite the majority of literature reporting cases of granulomatous hepatitis in immunocompromised patients [15-18], the workup of our patient was negative to tests that would indicate immunodeficiency, such as HIV, autoimmune, and chronic medical conditions.

Treatment is indicated for all patients with disseminated histoplasmosis. The management depends on disease severity and organ involvement and is typically with anti-fungal agents. Itraconazole should be administered three times daily for three days, and then daily maintenance treatment that is optimally continued for a year [19]. Amphotericin B can be used when there is severe disease with central nervous system involvement [20]. Fluconazole has not been found effective in the case of DH as itraconazole and is not used routinely for treatment [21]. Patients who received fluconazole developed resistance faster than with other antifungal agents [22]. In our case, the patient was continued on daily itraconazole as well as regular follow-ups, and his LFTs normalized nine months after treatment with itraconazole.

\section{Conclusions}

The diagnosis of liver involvement in histoplasmosis can be challenging, which may lead to inappropriate therapy or unnecessary interventions and liver biopsy may be considered in the presence of elevated liver function tests if the diagnosis was not achieved by less invasive testing as clinically indicated. Treatment with systematic antifungal medications is highly effective for these patients.

\section{Additional Information}

\section{Disclosures}

Human subjects: Consent was obtained or waived by all participants in this study. IRB approval was waived issued approval NA. IRB approval was waived and verbal consent was obtained from the patient. No identifying information appear in this article. Conflicts of interest: In compliance with the ICMJE uniform disclosure form, all authors declare the following: Payment/services info: All authors have declared that no financial support was received from any organization for the submitted work. Financial relationships: All authors have declared that they have no financial relationships at present or within the previous three years with any organizations that might have an interest in the submitted work. Other relationships: All authors have declared that there are no other relationships or activities that could appear to have influenced the submitted work.

\section{References}

1. Chu JH, Feudtner C, Heydon K, Walsh TJ, Zaoutis TE: Hospitalizations for endemic mycoses: a populationbased national study. Clin Infect Dis. 2006, 42:822-5. 10.1086/500405 
2. Bahr NC, Antinori S, Wheat LJ, Sarosi GA: Histoplasmosis infections worldwide: thinking outside of the Ohio River valley. Curr Trop Med Rep. 2015, 2:70-80. 10.1007/s40475-015-0044-0

3. Kauffman CA: Histoplasmosis: a clinical and laboratory update . Clin Microbiol Rev. 2007, 20:115-32. 10.1128/CMR.00027-06

4. Wheat LJ: Diagnosis and management of histoplasmosis. Eur J Clin Microbiol Infect Dis. 1989, 8:480-90. 10.1007/BF01964063

5. Kahi CJ, Wheat LJ, Allen SD, Sarosi GA: Gastrointestinal histoplasmosis. Am J Gastroenterol. 2005, 100:22031. 10.1111/j.1572-0241.2005.40823.x

6. Deepe GJ: Histoplasma capsulatum. Principles and Practice of Infectious Disease, 7th Edition. Mandell GL, Bennett JE, Dolin R (ed): Churchill Livingstone Elsevier, Philadelphia; 2010. 3305-13.

7. Zhou P, Sieve MC, Tewari RP, Seder RA: Interleukin-12 modulates the protective immune response in SCID mice infected with Histoplasma capsulatum. Infect Immun. 1997, 65:936-42. 10.1128/IAI.65.3.936-942.1997

8. Wheat LJ, Slama TG, Norton JA, Kohler RB, Eitzen HE, French ML, Sathapatayavongs B: Risk factors for disseminated or fatal histoplasmosis. Analysis of a large urban outbreak. Ann Intern Med. 1982, 96:159-63. 10.7326/0003-4819-96-2-159

9. Wahab NA, Mohd R, Zainudin S, Kamaruddin NA: Adrenal involvement in histoplasmosis. EXCLI J. 2013, $12: 1-4$.

10. Wheat LJ, Connolly-Stringfield PA, Baker RL, et al.: Disseminated histoplasmosis in the acquired immune deficiency syndrome: clinical findings, diagnosis and treatment, and review of the literature. Medicine (Baltimore). 1990, 69:361-74. 10.1097/00005792-199011000-00004

11. Goodwin RA Jr, Shapiro JL, Thurman GH, Thurman SS, Des Prez RM: Disseminated histoplasmosis: clinical and pathologic correlations. Medicine (Baltimore). 1980, 59:1-33.

12. Drebber U, Kasper HU, Ratering J, Wedemeyer I, Schirmacher P, Dienes HP, Odenthal M: Hepatic granulomas: histological and molecular pathological approach to differential diagnosis--a study of 442 cases. Liver Int. 2008, 28:828-34. 10.1111/j.1478-3231.2008.01695.x

13. Lanza FL, Nelson RS, Somayaji BN: Acute granulomatous hepatitis due to histoplasmosis . Gastroenterology. 1970, 58:392-6.

14. Rihana NA, Kandula M, Velez A, Dahal K, O'Neill EB: Histoplasmosis presenting as granulomatous hepatitis: case report and review of the literature. Case Rep Med. 2014, 2014:879535. 10.1155/2014/879535

15. Kothadia JP, Kone V, Giashuddin S: Granulomatous hepatitis: a rare primary manifestation of disseminated histoplasmosis in a renal transplant recipient. J Gastrointestin Liver Dis. 2017, 26:114. 10.15403/jgld.2014.1121.262.kot

16. Washburn L, Galván NT, Dhingra S, Rana A, Goss JA: Histoplasmosis hepatitis after orthotopic liver transplantation. J Surg Case Rep. 2017, 11:232. 10.1093/jscr/rjx232

17. Park S, Cheong J, Kyi K, et al.: Cholestasis and disseminated histoplasmosis in a psoriatic patient on infliximab: case report and review of literature. BMC Gastroenterol. 2020, 8:141. 10.1186/s12876-02001290-3

18. Pinheiro Bdo V, Delgado Ade A, Chebli JM: Hepatitis and pneumonitis during adalimumab therapy in Crohn disease: mind the histoplasmosis!. Arq Gastroenterol. 2014, 51:73-6. 10.1590/s0004-28032014000100015

19. Wheat LJ, Freifeld AG, Kleiman MB, Baddley JW, McKinsey DS, Loyd JE, Kauffman CA: Clinical practice guidelines for the management of patients with histoplasmosis: 2007 update by the Infectious Diseases Society of America. Clin Infect Dis. 2007, 45:807-25. 10.1086/521259

20. Johnson PC, Wheat LJ, Cloud GA, et al.: U.S. National Institute of Allergy and Infectious Diseases Mycoses Study Group. Safety and efficacy of liposomal amphotericin B compared with conventional amphotericin B for induction therapy of histoplasmosis in patients with AIDS. Ann Intern Med. 2002, 16:105-9. 10.7326/0003-4819-137-2-200207160-00008

21. McKinsey DS, Kauffman CA, Pappas PG, et al.: Fluconazole therapy for histoplasmosis. The National Institute of Allergy and Infectious Diseases Mycoses Study Group. Clin Infect Dis. 1996, 23:996-1001. 10.1093/clinids/23.5.996

22. Wheat LJ, Connolly P, Smedema M, Brizendine E, Hafner R: Emergence of resistance to fluconazole as a cause of failure during treatment of histoplasmosis in patients with acquired immunodeficiency disease syndrome. Clin Infect Dis. 2001, 33:1910-3. 10.1086/323781 